\title{
Video Article \\ Sublingual Immunotherapy as an Alternative to Induce Protection Against Acute Respiratory Infections
}

\author{
Natalia Muñoz-Wolf ${ }^{1,2}$, Analía Rial ${ }^{1}$, José M. Saavedra ${ }^{1}$, José A. Chabalgoity ${ }^{1}$ \\ ${ }^{1}$ Departamento de Desarrollo Biotecnológico, Universidad de la República \\ ${ }^{2}$ Present Affiliation: Trinity Biomedical Science Institute, Trinity College Dublin
}

Correspondence to: José A. Chabalgoity at jachabal@higiene.edu.uy

URL: https://www.jove.com/video/52036

DOI: doi:10.3791/52036

Keywords: Medicine, Issue 90, Sublingual immunotherapy, Pneumonia, Streptococcus pneumoniae, Lungs, Flagellin, TLR5, NLRC4

Date Published: 8/30/2014

Citation: Muñoz-Wolf, N., Rial, A., Saavedra, J.M., Chabalgoity, J.A. Sublingual Immunotherapy as an Alternative to Induce Protection Against Acute Respiratory Infections. J. Vis. Exp. (90), e52036, doi:10.3791/52036 (2014).

\section{Abstract}

Sublingual route has been widely used to deliver small molecules into the bloodstream and to modulate the immune response at different sites. It has been shown to effectively induce humoral and cellular responses at systemic and mucosal sites, namely the lungs and urogenital tract. Sublingual vaccination can promote protection against infections at the lower and upper respiratory tract; it can also promote tolerance to allergens and ameliorate asthma symptoms. Modulation of lung's immune response by sublingual immunotherapy (SLIT) is safer than direct administration of formulations by intranasal route because it does not require delivery of potentially harmful molecules directly into the airways. In contrast to intranasal delivery, side effects involving brain toxicity or facial paralysis are not promoted by SLIT. The immune mechanisms underlying SLIT remain elusive and its use for the treatment of acute lung infections has not yet been explored. Thus, development of appropriate animal models of SLIT is needed to further explore its potential advantages.

This work shows how to perform sublingual administration of therapeutic agents in mice to evaluate their ability to protect against acute pneumococcal pneumonia. Technical aspects of mouse handling during sublingual inoculation, precise identification of sublingual mucosa, draining lymph nodes and isolation of tissues, bronchoalveolar lavage and lungs are illustrated. Protocols for single cell suspension preparation for FACS analysis are described in detail. Other downstream applications for the analysis of the immune response are discussed. Technical aspects of the preparation of Streptococcus pneumoniae inoculum and intranasal challenge of mice are also explained.

SLIT is a simple technique that allows screening of candidate molecules to modulate lungs' immune response. Parameters affecting the success of SLIT are related to molecular size, susceptibility to degradation and stability of highly concentrated formulations.

\section{Video Link}

The video component of this article can be found at https://www.jove.com/video/52036/

\section{Introduction}

The overall goal of this work is to illustrate the benefits of sublingual immunotherapy for the treatment of acute respiratory infections (ARI) and present the advantages of this delivery route compared to other routes of administration, namely intranasal.

ARI cause millions of deaths every year especially in children under five. Streptococcus pneumoniae remains as one of the major etiological agents of bacterial pneumonia in infants and the elderly ${ }^{1,2}$. To present, the main available treatment relies on the use of antibiotics but resistant strains are continuously arising ${ }^{3,4}$.

SLIT induces broad responses at systemic and also mucosal level, particularly at the respiratory tract ${ }^{5}$. It has proven effectiveness against influenza infection, promoting long term protection with production of humoral and cellular responses ${ }^{6,7}$. Besides, it has been shown that prophylactic treatment with bacterial lysates delivered by sublingual route reduced exacerbations of chronic obstructive bronchitis in the elderly ${ }^{8}$ and prevented recurrent respiratory infections in children ${ }^{9}$. SLIT has been widely used for the treatment of allergies and asthma. Clinical studies had not only demonstrated its efficacy to modulate the immune response in the respiratory tract but also its safety ${ }^{10}$. Despite the growing interest of pharmaceutical companies and researchers in SLIT, the mechanisms involved in the induction of mucosal immune responses after sublingual delivery of compounds remain obscure. Recently, attention has been focused on the mechanisms promoting tolerance associated with allergen desensitization. It has been proposed that resident and recruited cells at the sublingual mucosa, like dendritic cells and macrophages, can promote tolerance after SLIT ${ }^{11-13}$. Dendritic cells of the oral mucosa can promote IFN-gamma and IL-10 producing T helper cells ${ }^{11}$ as well as recirculate to the distal genital mucosa and promote $\mathrm{CD}^{+} \mathrm{T}$ cells ${ }^{14}$. However, little is known about the impact of SLIT on innate cells or its capacity to improve pathogen clearance during acute respiratory infections.

The natural control of pneumococcal infection in the lungs greatly depends on the efficient and swift activation of local innate defences. We previously showed that enhancement of lungs' innate immunity by a single intranasal dose of flagellin (FliC), a TLR5 and NLRC4 agonist, 
protects $75-100 \%$ of mice challenged with a lethal dose of a clinical isolate of Streptococcus pneumoniae serotype 1. This protection was shown to be dependent on local recruitment of $\mathrm{GR} 1^{+}$cells (likely polymorphonuclear neutrophils, PMNs) and not dependent on antibodies, B or T cells ${ }^{15}$.

Flagellin is the structural component of the bacterial flagellum. In its monomeric form it is recognized by two Pathogen Recognition Receptors (PRRs), TLR5 that senses extracellular FliC ${ }^{16}$ and NLRC4/NAIP5 inflammasome that detects intracellular flagellin ${ }^{17,18}$. When FliC is sensed by the PRRs an important inflammatory response is triggered. We and others have demonstrated that instillation of purified FliC from Salmonella enterica serovar Typhimurium into the lungs drives swift production of chemokines and cytokines specially when recognized by the lungs' epithelium that in turn orchestrate the recruitment of immune cells into the airways, mainly PMNs ${ }^{15,19-21}$. Although transient, the substantial neutrophil infiltration that takes place into the airways after nasal delivery of FliC could be a concern if moving towards clinical therapies for human use. Excessive inflammation could be detrimental for the lungs' function. Moreover, it has been shown that intranasal delivery of immunostimulatory molecules may cause facial paralysis and/or brain toxicity ${ }^{22-24}$.

Sublingual immunotherapy offers a safer alternative to modulate the immune response in the respiratory tract compared to the intranasal route. It is non-invasive, painless, simple and has good patient compliance ${ }^{25}$. Furthermore, as mentioned before, it can induce protective responses in the respiratory mucosa without the risks associated to direct intranasal or intrapulmonary delivery of formulations. Sublingual route could be alternatively used to deliver molecules that have great effects onto the lung's immune system but that have been proven to be toxic or to elicit great inflammation when administered intranasally. Besides these advantages, formulations for sublingual immunotherapy have lower cost of manufacture since non-sterile products can be delivered by this route and endotoxic shock is not a concern for SLIT. On the other hand, it is worth noticing that higher doses of the immunostimulatory compounds compared to those used by intranasal or parenteral routes are necessary to induce an immune response in the lungs; also highly concentrated solutions are needed when using the mouse model of SLIT since the anatomical site where the formulations are deposited is small.

Based on our previous published data, we developed a model of protection using sublingual immunotherapy with flagellin as model immunostimulant. We demonstrated that a single dose of flagellin induced $60 \%$ survival against invasive pneumococcal pneumonia caused by the serotype 1 strain while all mice in the control group died of infection within 5 days. Flow cytometry analysis showed that higher numbers of $\mathrm{PMN}$ are recruited into the airways of protected animals after sublingual treatment with flagellin suggesting that these cells might be involved in the mechanism of protection induced by sublingual immunotherapy.

This video shows in detail how to perform sublingual immunotherapy and also how to recover relevant tissue from the sublingual mucosa, draining lymph nodes as well as lungs and airways to perform further analysis. Additionally, it illustrates the general technique of cell preparation for FACS analysis and briefly shows how to prepare Streptococcus pneumoniae suspensions and how to perform intranasal infections in mouse to set up the acute infection model.

\section{Protocol}

Procedures involving animals were performed in accordance with the protocols $N^{\circ} 071140-000821-12$ and 08052010 approved by the Honorary Commission for Animal Experimentation and the Directive Board of the School of Medicine, Universidad de la República - Uruguay.

\section{Sublingual Administration of the Therapeutic Agent}

1. Prepare the solution containing the therapeutic agent to be tested. Adjust concentration to administrate a maximum volume of $10 \mu \mathrm{l}$ per mouse.

NOTE: For purified flagellin from Salmonella enterica serovar Typhimurium the optimal dose to induce protection in mouse infected with the first lethal dose of $S$. pneumoniae serotype $1 \mathrm{E} 1586$, causing $100 \%$ mortality is $10 \mu \mathrm{g} / \mathrm{mouse}$. Flagellin solution must be heated at $65{ }^{\circ} \mathrm{C}$ for 5 $\min$ to ensure release of the monomers. For more information about flagellin purification see reference ${ }^{26}$.

1. Vary effective concentration of different immunomodulatory agents according to its molecular size, purity, susceptibility to proteolysis and the use of mucoadhesive agents. Adjust optimal concentration for each compound to be tested to maximize its effects. If previous studies by intranasal route have been conducted for a particular compound, use a starting dose 5 to 10 times higher to test its efficacy by sublingual route.

2. Anaesthetize the mice by injecting a cocktail containing $110 \mathrm{mg} / \mathrm{kg}$ Ketamine with $5.5 \mathrm{mg} / \mathrm{kg}$ Xylacine and let the animals rest for 7 to $10 \mathrm{~min}$.

3. Confirm proper anaesthetization by gently pressing the footpad of one of the hind legs; if properly anaesthetized the animal will not move in response to the stimulus.

4. Spread a thin layer of vet ointment over the eyes of each mouse to prevent dryness while under anaesthesia. NOTE: Inhalatory anesthetics like Isofluorane can be also used instead Ketamine/Xylacine if a system equipped with induction chamber and nose cones is available. Use the induction chamber to anaesthetise the animals and administrate the immunostimulant by sublingual route. Immediately connect the animal to a nose cone for at least $15 \mathrm{~min}$ to keep it under anaesthesia to avoid swallowing and allow absorption of the therapeutic compound.

5. Pipette the solution containing the immunostimulant or vehicle control solution; using the thumb and index finger of the non-dominant hand take the mouse and hold it in vertical position.

6. Using the dominant hand place a pair of closed forceps under the tongue and hold it in place using the middle and ring fingers, open the forceps slightly to lift the tongue.

7. Take the pipette and administer the solution onto the floor of the mouth and dorsal side of the tongue.

8. Remove the forceps and let the mouse rest for 3 to $5 \mathrm{~min}$ before putting it back into the cage. To ensure that normothermia is maintained in the anaesthetized mice, connect the cages to a cage heater system. If such system is not available, place mice belonging to the same treatment group back into the corresponding cage one next to each other over the bedding and partially cover them with clean tissue paper sheets to help them maintain the body temperature. 
9. Collect tissue samples at any time point after instillation of the immunomodulatory agent to analyze changes in the cell populations induced by the treatment.

NOTE: In this particular protocol administration of flagellin was performed $2 \mathrm{~h}$ before challenge. Determine optimal time between treatment and challenge for each particular therapeutic agent and pathogen to be tested.

\section{Preparation of the Bacterial Suspension and Intranasal Challenge with Streptococcus pneumoniae}

NOTE: S. pneumoniae is a natural human pathogen that can cause life threatening diseases like invasive pneumonia, sepsis and meningitis. Transmission may occur when inhaled or in contact with mucosa. Therefore, all samples that may have been in contact with S. pneumoniae must be handled in an appropriate Biosecurity Level II facility using a class II biosafety cabinet. Check the Standard Operating Procedures of your institution regarding handling of Type II pathogens for protective clothing, waste disposal and additional security measures that may apply. Infected animals should be kept in individually ventilated cages in isolators equipped with HEPA filters. Anti-pneumococcal vaccines and antibiotic therapy are available. For more information see references ${ }^{27}$ and ${ }^{1}$.

1. Thaw an aliquot of a working stock suspension of Streptococcus pneumoniae of known bacterial CFU number prepared as described in ${ }^{15}$.

2. Centrifuge for $5 \mathrm{~min}$ at $2,500 \mathrm{xg}$ and RT.

3. Discard the supernatant and wash the bacterial pellet by suspending it in $1 \mathrm{ml}$ of sterile saline solution. Use filter tips when preparing bacterial suspension, dilutions or for animal challenge.

4. Centrifuge again as described in step 2.2

5. Discard the supernatant and resuspend the pellet in the appropriate volume of sterile saline to obtain a suspension of $4 \times 10^{5} \mathrm{CFU} / 50 \mu \mathrm{Il}$. This dose corresponds to the minimum bacterial dose of $S$. pneumoniae serotype $1 \mathrm{E} 1586$ that causes $100 \%$ mortality in BALB/c mice according to previous studies ${ }^{15}$

NOTE: When establishing a model of pneumococcal pneumonia in mice, the minimum bacterial dose causing $100 \%$ of mortality must be determined for each particular combination of bacterial strain, serotype and mouse strain.

6. Homogenize the bacterial suspension by vortexing or pipetting up and down 5 times.

7. Load $50 \mu \mathrm{l}$ of the bacterial suspension using a sterile filter tip and instill the total volume into the nostrils of an anaesthetized mouse. Hold the mouse upright for $2 \mathrm{~min}$ and let it rest in dorsal position for 2 more min. Apply vet ointment onto the eyes and return the animals to the cage; make sure to maintain normothermia while under anaesthesia.

NOTE: In this study bacterial challenge was performed in a volume of $50 \mu$ to ensure delivery of at least $90 \%$ of the total CFU into the lungs as determined previously in ${ }^{15,28}$. To minimize distress of the animal's smaller volumes (e.g., $\left.20 \mu \mathrm{l}\right)$ can be used. However, efficient delivery of bacteria into the lungs must be checked; this can be done by harvesting the lungs 5 min after challenge and counting CFU in lungs' homogenates by plating serial dilutions onto blood-agar plates.

8. Confirm the CFU numbers in the bacterial suspension used for infection by plating serial 10 -fold dilutions onto blood agar plates. Incubate O/ $\mathrm{N}$ at $37^{\circ} \mathrm{C}$ with $5 \% \mathrm{CO}_{2}$ and count the number of mucoid colonies presenting a green halo characteristic of alpha haemolytic bacteria.

\section{Tissue Collection and Sample Preparation for Flow Cytometry (FACS) Analysis}

\section{1) Tissue collection}

1. Euthanize the animal by cervical dislocation or using a $\mathrm{CO}_{2}$ chamber; open the thoracic cavity all the way up to the neck and make an incision along the front legs to expose the ventral side of the neck and submandibular area.

2. With the fine tip curved forceps gently pull up the salivary glands and adjacent soft tissue to expose the dorsal side of the mouth floor. Using curved thin tip forceps, take the mandibular and accessory mandibular lymph nodes by pulling up gently and place them in a tube containing complete RPMI (cRPMI, for $500 \mathrm{ml}-10 \%$ Foetal Bovine Serum, $5 \mathrm{ml}$ of a solution containing 10,000 U/ml Penicillin and $10 \mathrm{mg} / \mathrm{ml}$ Streptomycin solution and $5 \mathrm{ml}$ of L-Glutamine $200 \mathrm{mM}$ ) or nucleic acid preservative solution according to the downstream procedure that will be carried out later.

3. To open the thoracic cavity make an incision in the diaphragm; using a pair of rat-toothed forceps clamp the xyphoid cartilage of the sternum and carefully cut the ribs at both dorsal sides starting from the false ribs all the way up until reaching the point where the true ribs meet the manubrium of the sternum.

4. By holding the xyphoid cartilage of the sternum with the forceps, pull up gently to expose the organs of the thoracic cavity.

5. Remove the ribs completely by cutting the first ribs and the clavicle. The thymus will appear as a white structure of two lobes located in the anteroventral portion of the thorax close to the base of the heart.

6. Take one of the lobes by clamping it with a pair of forceps and use a pair of scissors to remove the ligaments between its inferior face and the pericardium. Proceed to remove the second lobe.

7. Identify the abdominal cavity and open it by cutting along the median axis of the muscular wall to expose the organs. With a pair of forceps cut the posterior vena cava and thoracic aorta; remove the excess of blood with an absorbent tissue.

8. To analyse the resident and infiltrating cell populations of the alveoli perform bronchoalveolar lavage (BAL). Cut the muscles in the ventral part of the neck to expose the trachea and oesophagus; to separate them make incisions at lateral and dorsal sides of the structures.

9. Lift up the trachea with the forceps and make a small incision with a scalpel to introduce a thin-tip transfer pipette filled with $1 \mathrm{ml}$ of PBS without $\mathrm{Ca}^{2+} / \mathrm{Mg}^{2+}$ plus $1 \mathrm{mM}$ EDTA. Instil and aspirate the total volume at least three times; aspirate and transfer the cell suspension to a sterile $1.5 \mathrm{ml}$ tube and place it on ice.

10. To analyze the cell populations present in lung parenchyma, first perfuse the lungs by injecting $5 \mathrm{ml}$ of $\mathrm{PBS}$ without $\mathrm{Ca}^{2+} / \mathrm{Mg}^{2+} \mathrm{plus}^{1} \mathrm{mM}$ EDTA into the right ventricle of the heart.

NOTE: This will eliminate most of the red blood cells and immune cells present into the lungs' blood vessels. If perfusion was performed correctly, lungs colour will shift from pink to white. 
11. Isolate the heart from the lungs by clamping it from the base of the left ventricle and delicately cut the blood vessels with scissors to remove it completely. Take the perfused lungs and place them in cRPMI or nucleic acid preservative solution depending on the downstream analysis to be performed.

12. For analysis of the cell populations in the sublingual mucosa, isolate the head of the animal and remove the salivary glands and adjacent soft tissue if it has not been done in step 3.2.1.

13. Make an incision on each side of the mouth until reaching the mandible joint and separate the inferior jaw together with the tongue and floor of the mouth, using pins fix it on the dissection board. Pull up the tongue; using a scalpel make an incision where the base of the tongue meets the floor of the mouth until reaching the third molars to expose the sublingual mucosa.

14. Remove the tongue completely; take a $0.5 \mathrm{~mm}$ biopsy punch and place it next to the lower incisors. Cut from the gingival insertion of the sublingual tissue and press gently until the floor of the mouth has been cut out completely.

15. Repeat one more time now placing the biopsy punch close to the third molars to complete removal of the sublingual tissue. Place on a clean tube containing cRPMI or nucleic acid preservative.

\section{2) Sample preparation for FACS analysis.}

1. Transfer the lungs' tissue isolated from each mouse into a 24-well plate and mince them with a clean pair of scissors until obtaining small pieces of tissue of approximately $2 \mathrm{~mm}$. Add $1 \mathrm{ml}$ per well of digestion medium containing $30 \mathrm{mg}$ of type II Collagenase, $50 \mu \mathrm{g} \mathrm{DNAse-I}$ in 1 $\mathrm{ml}$ of RPMI without FBS. Pipette up and down five times and incubate at $37^{\circ} \mathrm{C}$ and $5 \% \mathrm{CO}_{2}$ for $40 \mathrm{~min}$.

1. For analysis of cell populations in the sublingual tissue, substitute the digestion medium in 3.2 .1 with one containing 2 units of Dispase, $30 \mathrm{mg}$ type II Collagenase, $50 \mu \mathrm{g}$ DNAse-I in $1 \mathrm{ml}$ of RPMI. Incubate the tissue collected from one mouse in $500 \mu \mathrm{l}$ of digestion medium for $20 \mathrm{~min}$ at $37^{\circ} \mathrm{C}$ in an orbital shaker at $50 \mathrm{rpm}$.

2. After incubation, pipette up and down up to 10 times or $30 \mathrm{sec}$ until most of the tissue has been disrupted. Filter the cell suspension though a $40 \mu \mathrm{m}$ sterile cell strainer and wash with $5 \mathrm{ml}$ of PBS supplemented with $5 \mathrm{mM}$ EDTA.

NOTE: Complete digestion of the extracellular matrix and fibrous tissue will not be achieved. However, longer incubation times in presence of collagenase and/or dispase or aggressive trituration are not recommended since it will result in increased cell death and destruction of extracellular proteins affecting the overall result of the FACS analysis.

3. Centrifuge at $400 \times \mathrm{g}, 5 \mathrm{~min}, 4^{\circ} \mathrm{C}$.

1. For analysis of the cell populations in BAL, centrifuge the cells at $400 \times \mathrm{g}, 5 \mathrm{~min}$, at $4{ }^{\circ} \mathrm{C}$ and continue to step 3.2.4.

2. For analysis of cell populations in the lymph nodes, place a $70 \mu \mathrm{m}$ cell strainer on a sterile petri dish and put the lymph nodes together with $1 \mathrm{ml}$ of cRPMI into the strainer. Take out the plunge of a $2 \mathrm{ml}$ sterile syringe and use it as a pestle to crush the lymph nodes against the strainer's mesh. Rinse the cell strainer with $1 \mathrm{ml}$ of fresh cRPMl and transfer the cells from the petri dish to a sterile tube.

4. Take a representative aliquot of each sample and stain it with Trypan Blue to determine viable cell number.

5. Resuspend the cells in FACS-EDTA: PBS- $5 \mathrm{mM}$ EDTA-1\% Bovine Serum Albumin- to make up a suspension of $2 \times 10^{7}$ cells $/ \mathrm{ml}$ and add $50 \mu \mathrm{l}$ into a cytometer tube.

6. Prepare a $2 X$ antibody mix containing the appropriate combinations of antibodies against surface markers and fluorochromes according to the available FACS instrument. Add $50 \mu \mathrm{l}$ of the $2 \mathrm{X}$ antibody mix into each tube containing the cell suspension. NOTE: Titrate each fluorochrome-labelled antibody to determine the optimal quantity to be used, for a detailed protocol see reference ${ }^{29}$.

7. Incubate 30 min on ice in the dark.

8. Wash one time with $3 \mathrm{ml}$ of FACS-EDTA and spin down the cells by centrifuging at $400 \mathrm{xg}$ for $5 \mathrm{~min}$ at $4^{\circ} \mathrm{C}$, resuspend the cells in $200 \mu \mathrm{l}$ of the same buffer and analyze in a Flow Cytometer.

NOTE: If handling a big number of samples, the staining protocol for FACS analysis described above can be performed in U-bottom 96-well plates instead of cytometer tubes. However, if using 96 -well plates washing steps must be performed by adding up to $200 \mu$ l of FACS-EDTA and repeating it 4 times spinning down the cells at $400 \mathrm{xg}$ for 5 min at $4{ }^{\circ} \mathrm{C}$ between each washing step.

9. At this point, fix the samples for analysis in the flow cytometer later (up to $72 \mathrm{~h}$ after fixation).

1. To fix the cells, after labelling with the FACS-antibodies wash the cells in PBS no $\mathrm{Ca}^{2+1} \mathrm{Mg}^{2+}, 1 \mathrm{mM}$ EDTA without FBS. Suspend the cells in $50 \mu \mathrm{l}$ of the same buffer and add $50 \mu \mathrm{l}$ of a freshly prepared $4 \%$ paraformaldehyde solution in hypertonic (2X) PBS no $\mathrm{Ca}^{2+1} \mathrm{Mg}^{2+}$

2. Incubate for 20 min at RT and wash 3 times in FACS-EDTA.

3. Resuspend the cells in $200 \mu$ of FACS-EDTA and store at $4{ }^{\circ} \mathrm{C}$ and protected from the light for up to $72 \mathrm{~h}$.

NOTE: FSC-SSC can be affected by fixation. If fixing the samples check compatibility of fluorescently labelled antibodies with the manufacturer since tandem dyes can be degraded in presence of fixative agents. If samples originated from infected animals fixation is highly recommended to ensure that no viable pathogens will be present when analysing the samples in the FACS machine since microaerosols can be generated during acquisition of the sample.

\section{Total RNA Extraction, cDNA Synthesis and Real Time PCR.}

\section{1) RNA extraction and cDNA synthesis.}

1. Homogenize the tissue in the nucleic acid preservative solution of choice by mechanical disruption (e.g., using a rotor-stator homogenizer, high speed shaking tissue-ruptor and beads, etc.). Centrifuge at $12,600 \times \mathrm{g}$ for $15 \mathrm{~min}$ and $4{ }^{\circ} \mathrm{C}$ to remove the tissue debris. Transfer the supernatant to a clean tube.

2. Extract the RNA with the method of choice following manufacturer instructions.

NOTE: RNA is highly susceptible to degradation, if it is not going to be used straight away after isolation, make aliquots and store them in RNAse free tubes at $-80^{\circ} \mathrm{C}$. Avoid repeated freezing and thawing. The tubes must be handled with gloves at all times. After thawing the samples always keep them on ice.

3. Measure the absorbance of nucleic acids at $260 \mathrm{~nm}$ and calculate the concentration in $\mu \mathrm{g} / \mu \mathrm{l}$. 
4. Prepare DNAse-I mix by addition of (for 1 sample): $7.6 \mu$ l of ultrapure water, $1 \mu \mathrm{l}$ of 10X DNAse-I buffer, $0.4 \mu \mathrm{l}$ of DNAse-I (amplification grade) stock $1 \mathrm{U} / \mu \mathrm{l}$, and add $8.4 \mu \mathrm{l}$ of the DNAse-I mix to each sample containing $1 \mu \mathrm{g}$ of total RNA.

1. Use RNA at a concentration of $1 \mu \mathrm{g} / \mu \mathrm{l}$ and perform the retrotranscription reaction (RT-PCR) by adding $1 \mu \mathrm{l}$ of the total RNA as a template. If the samples are too diluted and the concentration is lower than expected, add larger volumes of total RNA instead of water. Do not exceed $20 \%$ of the final reaction volume when adding the RNA specially if the RNA extraction protocol of choice involved phenol-chloroform mixture since phenol traces could affect the yield of the RT-PCR.

5. Incubate $15 \mathrm{~min}$ at RT followed by $10 \mathrm{~min}$ at $4{ }^{\circ} \mathrm{C}$ or ice. (Do not exceed the incubation time!!)

6. Add $1 \mu \mathrm{l}$ of EDTA $25 \mathrm{mM}$ (molecular biology grade) to each tube and incubate at $65^{\circ} \mathrm{C}$ for 10 min to inactivate the DNAse-I.

7. Prepare retrotranscription (RT) mix as follows (for 1 reaction): $1 \mu$ random hexamer primers stock $0.2 \mathrm{mg} / \mathrm{ml}, 1 \mu \mathrm{dNTPs}$ stock $10 \mathrm{mM}, 4 \mu \mathrm{l}$ 5X M-MLV-RT buffer, $2 \mu \mathrm{l}$ DTT $0.1 \mathrm{M}, 1 \mu \mathrm{l}$ RNAse OUT stock $40 \mathrm{U} / \mu \mathrm{l}$, and $1 \mu \mathrm{l} \mathrm{M-MLV} \mathrm{retrotranscriptase} \mathrm{stock} 200 \mathrm{U} / \mu \mathrm{l}$.

8. Add $10 \mu$ l of RT-PCR mix to the $10 \mu$ DNAse-I reaction tube.

9. Carry out the $\mathrm{PCR}$ reaction in a thermal cycler according to the following program: 1X cycle: $10 \mathrm{~min}, 25^{\circ} \mathrm{C} ; 50 \mathrm{~min}, 37^{\circ} \mathrm{C} ; 15 \mathrm{~min}, 70^{\circ} \mathrm{C}$

10. Dilute the cDNA $1: 5$ by adding $80 \mu$ l of ultrapure water. Store at $-20^{\circ} \mathrm{C}$.

\section{2) Real time PCR (qPCR).}

1. Prepare qPCR reaction mix as follows (for 1 reaction): $5 \mu$ master mix containing Taq DNA Polymerase, SYBR Green dye, PCR Buffer, dNTP mix and $\mathrm{MgCl}_{2}$ (see 4.2.2 below); $0.9 \mu \mathrm{l}$ of a $10 \mu \mathrm{M}$ stock solution of the forward primer, $0.9 \mu \mathrm{l}$ of a $10 \mu \mathrm{M}$ stock solution of the reverse primer, $1.2 \mu \mathrm{l}$ ultrapure water, and $2 \mu \mathrm{l}$ of cDNA template previously diluted as indicated in step 4.1.10.

NOTE: Reagent concentration and cycling protocols used in this section were optimised to be carried out specifically with the reagents and instruments described in "Table of Materials and Reagents", other brands can be used but reaction volumes, reagent concentration and cycling protocol may vary. Check your manufacturer instructions before performing RT-qPCR.

2. Set up the qPCR instrument as follows:

1X cycle: $15 \mathrm{~min}, 95^{\circ} \mathrm{C}$

40X cycles: $15 \mathrm{sec}, 95^{\circ} \mathrm{C}$ followed by $1 \mathrm{~min}, 60^{\circ} \mathrm{C}$ (at this point acquire fluorescence).

NOTE: For relative quantification of mRNA according to the $\mathrm{Ct}$ method ${ }^{30}$ a reference gene must be selected for normalization of the $\mathrm{Ct}$ values. Reference gene of choice should be tested under specific assay conditions as its expression may vary; Actb, Gapdh or 18S are some of the genes usually selected as references.

3. Set up the threshold value and analyze the data.

\section{Representative Results}

Sublingual immunotherapy can be successfully used to modulate lungs' immune response. We showed that a single dose of flagellin, the TLR5 and NLRC4 agonist, can induce significant upregulation of the mRNA encoding the chemokines CXCL1, CCL20 and the cytokine IL-6 compared to saline treated controls. Fold induction of mRNA levels peaked at $8 \mathrm{~h}$ after SLIT and return to basal levels after $20 \mathrm{hr}$ (Figure 1). However, when SLIT was performed $2 \mathrm{hr}$ prior intranasal infection with S. pneumoniae, levels of Cxcl1 and $I / 6$ mRNA remained significantly upregulated even $24 \mathrm{hr}$ after SLIT compared to non-treated animals (Figure 2).

Analysis of the cell populations in BAL and lung tissue by FACS revealed that animals treated with FliC by sublingual route had increased number of neutrophils in the airways but not in the lungs' tissue (Figure 3 ).

Finally, survival after pneumococcal challenge was compared in animals previously treated with FliC by sublingual route or with saline as a control. As shown in Figure 4, SLIT with flagellin promoted protection and increased survival against acute pneumococcal pneumonia. 


\section{Cxcl1}

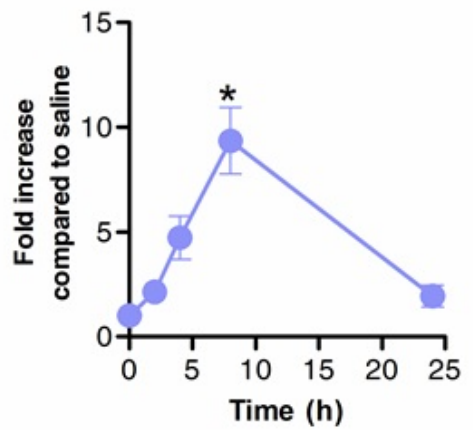

$\mathrm{Ccl} 20$

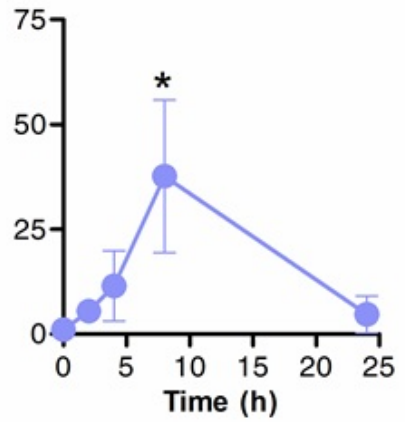

116

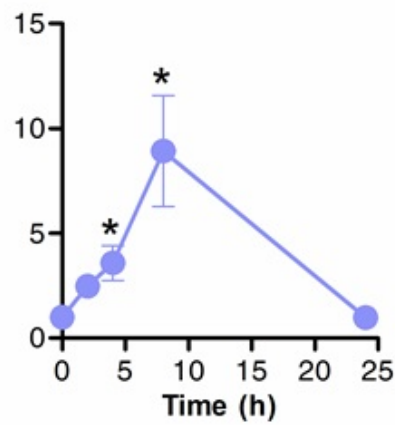

Figure 1. Kinetics of the lungs' transcriptional profile after sublingual immunotherapy with flagellin. Eight to 10 weeks old BALB/C mice $(n=4)$ were treated with $10 \mu \mathrm{g}$ of flagellin or saline by sublingual route under anaesthesia. Lungs were collected at different time points and placed in nucleic acid preservative. Total RNA extraction was performed and cDNA was synthesized. mRNA levels were evaluated by real time PCR using specific primers listed in Table 1. Relative quantification was performed according $\triangle$ Ct method using Actb mRNA levels for normalization. Results are shown as fold increase compared to saline treated group as median \pm SEM. Asterisks indicate statistically significant differences $(p<0.05)$ calculated according to Mann-Whitney test. Results are representative of 2 independent experiments.

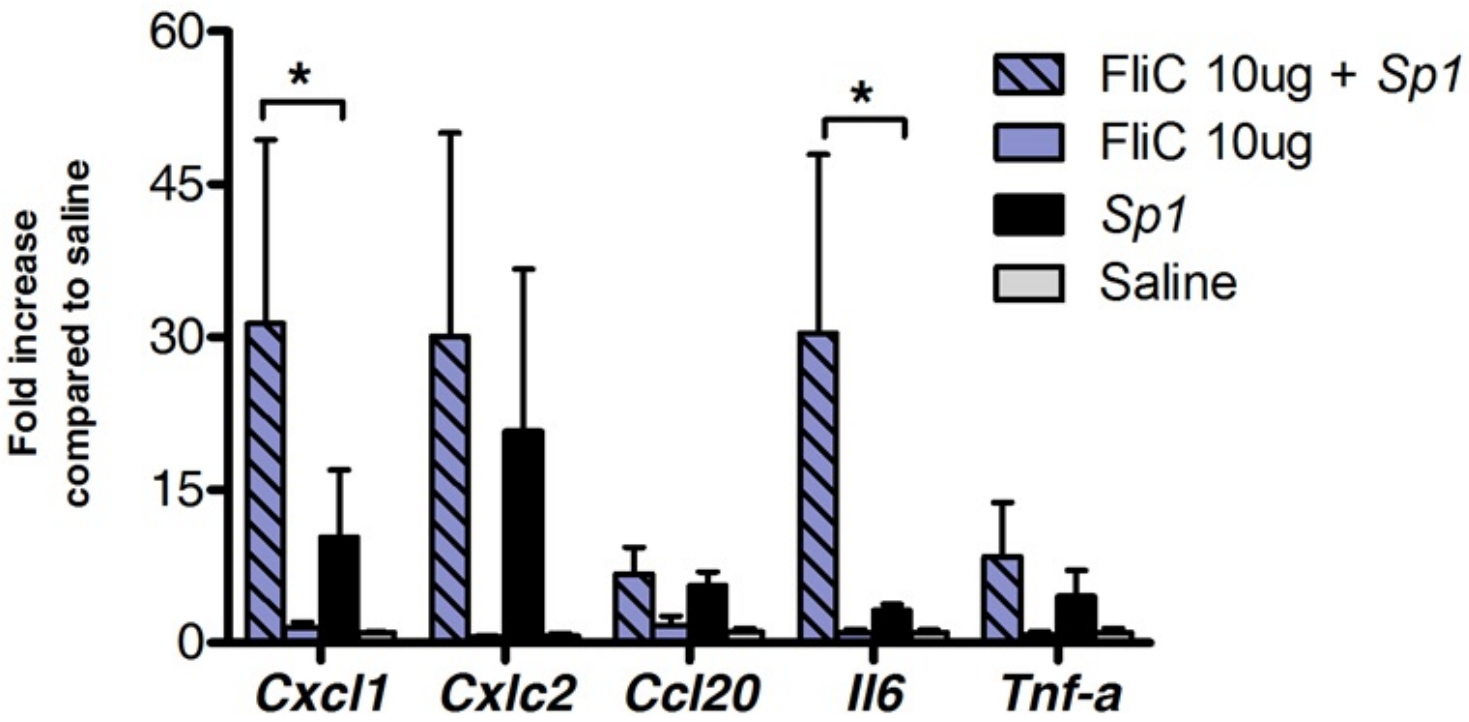

Figure 2. Lungs' transcriptional profile during pneumococcal pneumonia after sublingual immunotherapy with flagellin. Eight to 10 weeks old BALB/c mice ( $n=4$ for control group and $n=7$ for treated group) were treated with $10 \mu \mathrm{g}$ of flagellin or saline by sublingual route under anaesthesia. $2 \mathrm{hr}$ later mice were challenged by intranasal route with the minimal lethal dose (MLD) causing $100 \%$ mortality of a clinical isolate of S. pneumoniae serotype $1 \mathrm{E} 1585$, corresponding to $4 \times 10^{5} \mathrm{CFU} / 50 \mu \mathrm{l}$. Lungs were collected $24 \mathrm{hr}$ after challenge and stored in nucleic acid preservative until RNA extraction and cDNA synthesis were carried out. Real time PCR was carried out (See primer list in Table 1) and relative quantification was performed according $\triangle$ Ct method using Actb mRNA levels for normalization. Results are shown as fold increase compared to saline treated group as median \pm SEM. Asterisks indicate statistically significant differences $(p<0.05)$ calculated according to Mann-Whitney test. 


\section{PMN recruitment in BAL}

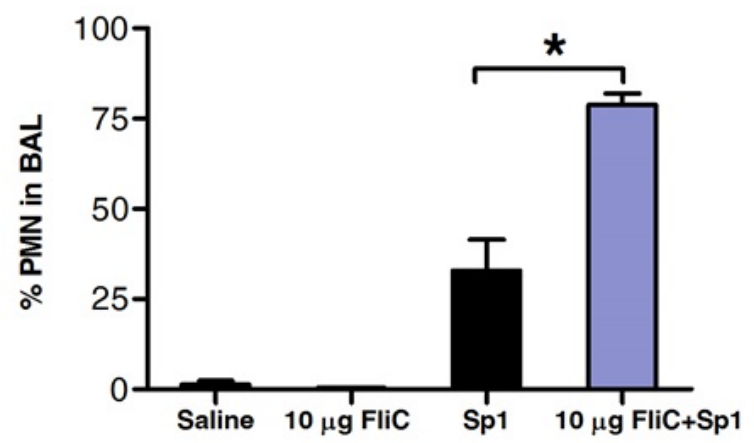

PMN recruitment in lungs

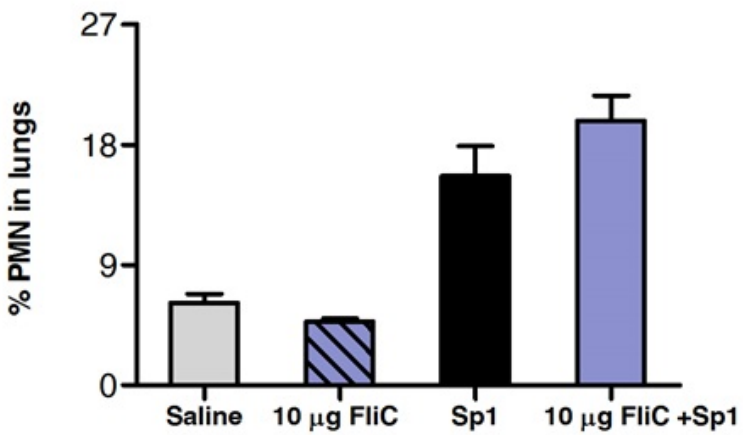

Figure 3. Analysis of polymorphonuclear neutrophil (PMN) recruitment in lungs' tissue and airways after SLIT. Eight to 10 weeks old BALB/c mice $(n=4)$ were treated with $10 \mu \mathrm{g}$ of flagellin or saline by sublingual route under anaesthesia. $2 \mathrm{hr}$ later mice were challenged by intranasal route with the MLD of $S$. pneumoniae serotype $1 \mathrm{E} 1585.24 \mathrm{hr}$ after challenge, BAL was performed and lungs were processed for FACS analysis. PMN were identified as Ly6G ${ }^{\text {high }} / C D 11 b^{\text {high }} / C D 11 c^{\text {negative }}$ cells and based on the FCS-SSC profile. Results are expressed as percentage of PMN with respect of total cell numbers in BAL or lungs. Bars represent median $\pm S E M$. Asterisks indicate statistically significant differences $(p<0.05)$ calculated according to one-way Mann-Whitney test.

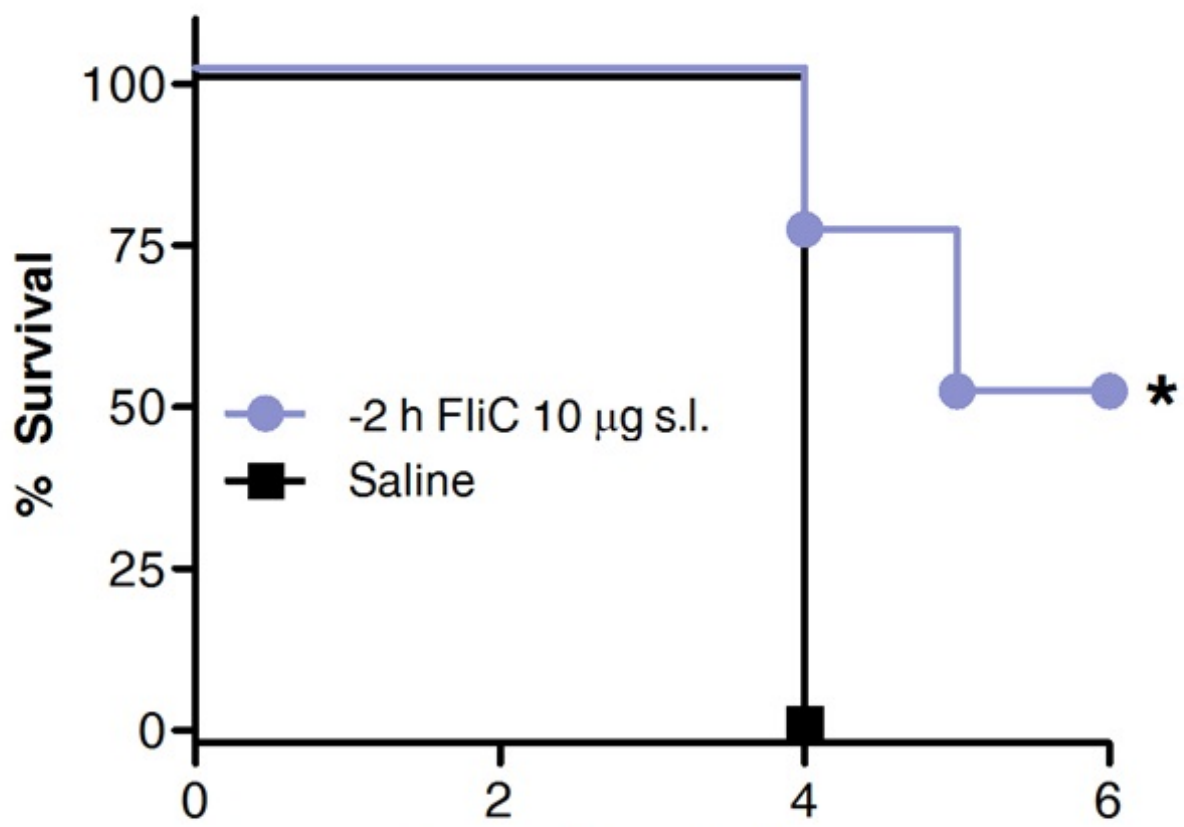

\section{Days after challenge}

Figure 4. SLIT with flagellin protects mice against acute pneumococcal pneumonia. Eight to 10 weeks old BALB/c mice $(n=8)$ were treated with $10 \mu \mathrm{g}$ of flagellin or saline by sublingual route under anaesthesia. $2 \mathrm{hr}$ later mice were challenged by intranasal route with the MLD of S. pneumoniae serotype 1 E1585. Survival was assessed on a daily basis. Kaplan-Meier curves were compared according Log-rank (MantelCox) test. Asterisks indicate statistically significant differences $(p<0.05)$. Results are representative of 2 independent experiments.

\begin{tabular}{|c|c|c|}
\hline Name & Sequence 5'-3' & PCR Product lenght (bp) \\
\hline mB-actin_F & GCTTCTTTGCAGCTCCTTCGT & \multirow[t]{2}{*}{68} \\
\hline$m B$-actin_R & CGTCATCCATGGCGAACTG & \\
\hline mCCL20_F & TTTTGGGATGGAATTGGACAC & \multirow[t]{2}{*}{69} \\
\hline mCCL20_R & TGCAGGTGAAGCCTTCAACC & \\
\hline
\end{tabular}




\begin{tabular}{|c|c|c|}
\hline mCXCL1_F & CTTGGTTCAGAAAATTGTCCAAAA & \multirow[t]{2}{*}{84} \\
\hline$m C X C L 1 \_R$ & ACGGTGCCATCAGAGCAGTCT & \\
\hline mIL-6_F & GTTCTCTGGGAAATCGTGGAAA & \multirow[t]{2}{*}{78} \\
\hline$m I L-6 \_R$ & AAGTGCATCATCGTTGTTCATACA & \\
\hline mTNFalpha_F & САTCTTCTCAAAATTCGAGTGACAA & \multirow[t]{2}{*}{63} \\
\hline mTNFalpha_R & CCTCCACTTGGTGGTTTGCT & \\
\hline$m C x c / 2 \_F$ & CCCTCAACGGAAGAACCAAA & \multirow[t]{2}{*}{72} \\
\hline$m C x c / 2 \_R$ & CACATCAGGTACGATCCAGGC & \\
\hline
\end{tabular}

Table 1. Primer list used for real time PCR analysis. Specific primer sequences used for $q P C R$ analysis. Forward and reverse primers for mouse actb, Cccl20, Cxcl1, II6, Tnfa and Cxcl1 are presented as 5'-3' sequences and expected product length is indicated in base pairs (bp).

\section{Discussion}

Sublingual administration of therapeutic agents has been proven as a useful means to modulate the immune response in the respiratory tract. The main advantage of SLIT for the treatment of respiratory conditions is that it does not involve direct delivery of compounds into the lungs or nostrils, being safer than treatments based on intranasal administration ${ }^{31}$.

Sublingual immunotherapy can be used to modulate the immune response in different ways, either for induction of regulatory responses that can ameliorate the symptoms of allergic inflammation and asthma ${ }^{32}$ or to induce transient activation of innate immune mechanisms to treat acute lung infections as shown here.

The mouse model presented in this video is a convenient method for screening of different compounds as therapeutic agents for SLIT.

This animal model offers a useful means to determine the impact of SLIT in the lungs' immune response as well as in other organs (e.g., draining lymph nodes or distal mucosal sites) that cannot be mimicked by the use of in vitro models. Although there are several papers that describe results obtained using sublingual immunotherapy, detailed methods for the procedures of sublingual administration have not been made available yet. Additionally, the model can be used for evaluation of sublingual vaccines aiming to confer systemic as well as local protection in the respiratory tract.

As shown in the accompanying video, sublingual administration of compounds is a simple procedure that can be easily performed without the need of extensive training. Typically, a person proficient in animal handling will require $1 \mathrm{hr}$ to perform SLIT in a group of 10 mice using injectable anesthetics as described in this protocol. If pneumococcal challenge is performed as well, 90 additional min will be required to prepare the bacterial suspension and perform intranasal challenge of the animals.

The FACS protocols presented here allow convenient characterisation of impact of the SLIT at the local site of administration, draining lymph nodes as well as their effects on the lungs' cell dynamics.

Separate analysis of the bronchoalveolar content and lung parenchyma is important to discriminate the airways' immune resident and infiltrating cell types from those that remain within the tissue. Analysis of the BAL content allows the study of alveolar macrophage turnover as well as the dynamics of cells recruitment into the alveolar spaces induced by different treatments, e.g., PMNs, eosinophils, monocytes. BAL can also be used to assess presence of secreted cytokines and chemokines by Enzyme-linked Immunosorbent Assay (ELISA) or detection of secreted IgA antibodies elicited after sublingual vaccination. Study of the lungs' tissue will allow characterisation of other cell types, classically dendritic cells, $T$ cells and $B$ cells.

Preparation of BAL samples and lymph nodes for FACS analysis is simple. After sample collection, normally 60 min are required to complete the staining protocol for 10-20 samples. In contrast, isolation of cells from lungs or sublingual tissue will require more time since digestion of the extracellular matrix is required. Absorption of the therapeutic agent delivered by sublingual route can be addressed by tracking of fluorescently or radioactively labelled molecules using in vivo imaging systems.

Sublingual immunotherapy is an attractive method to effectively induce immune responses in the respiratory tract as well as systemically that can be used to treat or prevent respiratory conditions. Elucidation of the mechanisms determining activation vs tolerance of the immune response in the respiratory tract after SLIT is crucial to allow rational design of new therapeutic strategies that could be used alone or in combination with available treatments against different respiratory conditions.

\section{Disclosures}

The authors have nothing to disclose.

\section{Acknowledgements}

We acknowledge Dr. Jean-Claude Sirard from the Center for Infection and Immunity of Lille, Institute Pasteur de Lille-France, for kindly providing the purified flagellin and Dr. Teresa Camou, Director of the National Reference Laboratory, Ministry of Health of Uruguay for kindly providing the pneumococcal strain. 
The authors would like to express their acknowledgement to Mr. Diego Acosta and Mr. Ignacio Turel form BichoFeo Producciones-Uruguay for their commitment and hard work during the entire video production and edition.

This work was supported by the grants PR_FCE_2009_1_2783 and BE_POS_2010_1_2544 from the National Agency of Research and Innovation, ANII from Uruguay, the Program for Development of Basic Sciences, PEDECIBA of Uruguay and Sectoral Commission of Scientific research, CSIC-Universidad de la República, Uruguay.

\section{References}

1. Pneumococcal vaccines WHO position paper--2012. Weekly Epidemiological Record. 14, 129-144 (2012).

2. Pneumonia - Facts Sheet $N^{\circ} 331$. WHO. http://www.who.int/mediacentre/factsheets/fs331/en/\# (2013).

3. Appelbaum, P. C., et al. Carriage of antibiotic-resistant Streptococcus pneumoniae by children in eastern and central Europe-a multicenter study with use of standardized methods. Clin Infect Dis. 23, 712-717 (1996).

4. Ramirez, J. A., Anzueto, A. R. Changing needs of community-acquired pneumonia. J Antimicrob Chemother. 66, 3-9, doi:10.1093/jac/dkr094 (2011).

5. Cuburu, N., et al. Sublingual immunization induces broad-based systemic and mucosal immune responses in mice. Vaccine. 25, 8598-8610, doi:10.1016/j.vaccine.2007.09.073 (2007).

6. Pedersen, G. K., et al. Evaluation of the sublingual route for administration of influenza H5N1 virosomes in combination with the bacterial second messenger c-di-GMP. PLoS One. 25, 1-12, doi:10.1371/journal.pone.0026973 (2011).

7. Song, J. H., et al. Sublingual vaccination with influenza virus protects mice against lethal viral infection. Proc Natl Acad Sci USA. 105, 1644-1649, doi:10.1073/pnas.0708684105 (2008).

8. Cogo, R., Ramponi, A., Scivoletto, G., Rippoli, R. Prophylaxis for acute exacerbations of chronic bronchitis using an antibacterial sublingual vaccine obtained through mechanical lysis: a clinical and pharmacoeconomic study. Acta Biomed. 74, 76-87 (2003).

9. Rosaschino, F., \& Cattaneo, L. Strategies for optimizing compliance of paediatric patients for seasonal antibacterial vaccination with sublingually administered Polyvalent Mechanical Bacterial Lysates (PMBL). Acta Biomed. 75, 171-178 (2004).

10. Senna, G., Caminati, M., \& Canonica, G. W. Safety and tolerability of sublingual immunotherapy in clinical trials and real life. Curr Opin Allergy Clin Immunol. 13, 656-662, doi:10.1097/ACI.0000000000000007 (2013).

11. Mascarell, L., et al. Oral dendritic cells mediate antigen-specific tolerance by stimulating TH1 and regulatory CD4+ T cells. J Allergy Clin Immunol. 122, 603-609 e605, doi:10.1016/j.jaci.2008.06.034 (2008).

12. Mascarell, L., et al. Mapping of the lingual immune system reveals the presence of both regulatory and effector CD4+ T cells. Clin Exp Allergy. 39, 1910-1919, doi:10.1111/j.1365-2222.2009.03337.x (2009).

13. Mascarell, L., et al. Oral macrophage-like cells play a key role in tolerance induction following sublingual immunotherapy of asthmatic mice. Mucosal Immunology. 4, 638-647, doi:10.1038/mi.2011.28 (2011).

14. Hervouet, C., et al. Antigen-bearing dendritic cells from the sublingual mucosa recirculate to distant systemic lymphoid organs to prime mucosal CD8 T cells. Mucosal Immunology. 7, 280-291, doi:10.1038/mi.2013.45 (2014).

15. Munoz, N., et al. Mucosal administration of flagellin protects mice from Streptococcus pneumoniae lung infection. Infect Immun. $\mathbf{7 8}$, 4226-4233, doi:10.1128/IAI.00224-10 (2010).

16. Hayashi, F., et al. The innate immune response to bacterial flagellin is mediated by Toll-like receptor 5. Nature. 410, 1099-1103, doi:10.1038/35074106 (2001).

17. Lightfield, K. L., et al. Critical function for Naip5 in inflammasome activation by a conserved carboxy-terminal domain of flagellin. Nature Immunology. 9, 1171-1178, doi:10.1038/ni.1646 (2008).

18. Lightfield, K. L., et al. Differential requirements for NAIP5 in activation of the NLRC4 inflammasome. Infect Immun. 79, 1606-1614, doi:10.1128/IAI.01187-10 (2011).

19. Honko, A. N., \& Mizel, S. B. Mucosal administration of flagellin induces innate immunity in the mouse lung. Infect Immun. 72, 6676-6679 (2004).

20. Janot, L., et al. Radioresistant cells expressing TLR5 control the respiratory epithelium's innate immune responses to flagellin. Eur $\mathrm{J}$ Immunol. 39 (6), 1587-96, doi:10.1002/eji.200838907 (2009).

21. Van Maele, L., et al. TLR5 signaling stimulates the innate production of IL-17 and IL-22 by CD3(neg)CD127+ immune cells in spleen and mucosa. J Immunol. 185, 1177-1185, doi:10.4049/jimmunol.1000115 (2010).

22. Lee, S. J., et al. Neurologic adverse events following influenza A (H1N1) vaccinations in children. Pediatrics international: official journal of the Japan Pediatric Society. 54, 325-330, doi:10.1111/j.1442-200X.2012.03568.x (2012).

23. Lewis, D. J., et al. Transient facial nerve paralysis (Bell's palsy) following intranasal delivery of a genetically detoxified mutant of Escherichia coli heat labile toxin. PLoS One. 4, e6999, doi:10.1371/journal.pone.0006999 (2009).

24. Mutsch, M., et al. Use of the inactivated intranasal influenza vaccine and the risk of Bell's palsy in Switzerland. N Engl J Med. 350, 896-903, doi:10.1056/NEJMoa030595 (2004).

25. Kuo, C. H., Wang, W. L., Chu, Y. T., Lee, M. S., \& Hung, C. H. Sublingual immunotherapy in children: an updated review. Pediatr Neonatol. 50, 44-49, doi:10.1016/S1875-9572(09)60031-0 (2009).

26. Nempont, C., Cavet, D., Rumbo, M., Bompard, C., Villeret, V., Sirard, J.C. Deletion of flagellin's hypervariable region abrogates antibodymediated neutralization and systemic activation of TLR5-dependent immunity. J. Immunol. 181, 2036-2043 (2008).

27. Pathogen Regulation Directorate, Public Health Agency of Canada. Streptococcus pneumoniae: Pathogen Safety Data Sheet - Infectious Substances. http://www.phac-aspc.gc.ca/lab-bio/res/psds-ftss/streptococcus-pneumoniae-eng.php\#footnote5 (2011).

28. Marques, J. M., et al. Protection against Streptococcus pneumoniae serotype 1 acute infection shows a signature of Th17- and IFN-gammamediated immunity. Immunobiology. 217, 420-429, doi:10.1016/j.imbio.2011.10.012 (2012).

29. Stewart, C. C., \& Stewart, S. J. Chapter 4, Titering antibodies. Current Protocols in Cytometry. Unit 4.1, doi:10.1002/0471142956.cy0401s14 (2001).

30. Kubista, M., et al. The real-time polymerase chain reaction. Molecular Aspects of Medicine. 27, 95-125, doi:10.1016/j.mam.2005.12.007 (2006).

31. Pedersen, G., \& Cox, R. The mucosal vaccine quandary: intranasal vs. sublingual immunization against influenza. Human Vaccines \& Immunotherapeutics. 8, 689-693, doi:10.4161/hv.19568 (2012). 
32. Vitaliti, G., et al. Mucosal immunity and sublingual immunotherapy in respiratory disorders. Journal of Biological Regulators and Homeostatic Agents. 26, S85-93 (2012). 